\title{
Desinformación: retos profesionales para el sector de la comunicación
}

\author{
Disinformation: Professional challenges for the \\ communication sector
}

\author{
Leticia Rodríguez-Fernández
}

Cómo citar este artículo:

Rodríguez-Fernández, Leticia (2019). “Desinformación: retos profesionales para el sector de la comunicación". El profesional de la información, v. 28, n. 3, e280306.

https://doi.org/10.3145/epi.2019.may.06

Artículo recibido el 13-12-2018

Aceptación definitiva: 28-05-2019

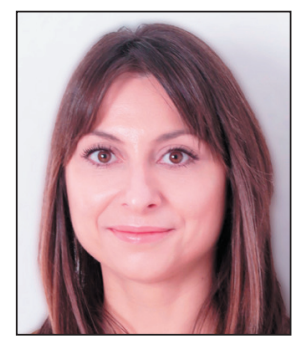

\author{
Leticia Rodríguez-Fernández $\square$ \\ https://orcid.org/0000-0002-7472-5472 \\ Universidad Nebrija \\ Santa Cruz de Marcenado, 27. 28015 Madrid, España \\ Irodrigu@nebrija.es
}

\section{Resumen}

El auge de la desinformación propicia el debate sobre su impacto en la sociedad y las posibilidades y herramientas para luchar contra ella. Los profesionales de la comunicación se encuentran ante nuevos contextos que afectan a la credibilidad de su trabajo. Este estudio trata de conocer cómo perciben el fenómeno los expertos, identificando posibles cambios en sus rutinas de trabajo. Se realizaron 24 entrevistas estructuradas a consultores políticos, verificadores de información, periodistas y responsables de redes sociales de medios digitales. Se evidencia que los cambios en los protocolos de trabajo resultan aún menores, aunque todos los actores sociales se hayan visto directamente afectados por el impacto de la desinformación. Tampoco hay consenso en torno a la creación de un posible marco legislativo que la aborde. Los resultados pueden ayudar a enfocar nuevas investigaciones en este campo, así como ofrecer una aproximación al estado actual de los profesionales de la comunicación ante el fenómeno.

\section{Palabras clave}

Desinformación; Posverdad; Fake news; Noticias falsas; Comunicación política; Verificación; Fact-checking; Profesionales de la comunicación; Consultores políticos; Periodistas; Verificadores.

\begin{abstract}
The rise of disinformation increases debate about its impact on society and the possibilities and tools to fight it. Communication professionals are faced with new contexts that affect the credibility of their work. This study tries to know how experts perceive the phenomenon by identifying possible changes in their work routines. Twenty-four structured interviews were conducted with political consultants, information verifiers, journalists and digital media social network managers. It was evidenced that the changes in working protocols are still small, although all social actors have been directly affected by the impact of disinformation. There is no consensus neither on the creation of a possible legislative framework to address it. The results can help to focus new research on this field as well as offer an approximation to the current state of communication professionals in face of the phenomenon.
\end{abstract}

\section{Keywords}

Disinformation; Post-truth; Fake news; Junk news; Political communication; Fact-checking; Communication professionals; Political consultants; Journalists; Fact-checkers. 


\section{Posverdad: lenguaje y burbujas}

El término posverdad (post-truth) fue utilizado por primera vez en 1992 por el escritor Steve Tesich en la revista Nation al referirse a varios casos polémicos en la política estadounidense, como el Watergate o la Guerra de Vietnam, en los que se había mentido deliberadamente a la opinión pública (Krienter, 2016). Se planteaba que la ciudadanía prefería vivir en un mundo de posverdad antes que enfrentarse a la realidad, justificando las mentiras de los políticos como un mecanismo de protección. Keyes (2004, p. 12) amplía el concepto a "la era de posverdad" y expone que el individuo no quiere pensar que no es ético y mucho menos admitirlo ante los demás. Por ello se idean enfoques alternativos que denomina "alt.ethics" (2004, p. 13) en los que se acepta la simulación como algo correcto. La lengua es el vehículo principal en la construcción de la posverdad, a través de lo que el autor denomina euphemasia (2004, p. 15). Esta tergiversación del lenguaje ofrece nuevas visiones de los hechos. Pérez (2017) señala que la mentira se renueva a través de la neolengua que resulta políticamente correcta, profundamente falaz, simple y eficaz. Se busca la apelación emocional, la aceptación de la mentira bajo marcos de creencias. Para el Oxford English Dictionary la posverdad

"denota circunstancias en que los hechos objetivos influyen menos en la formación de la opinión pública, que los llamamientos a la emoción y a la creencia personal" (Oxford English Dictionary, 2016).

En 2010 el periodista David Roberts ahonda en el término al referirse a los políticos de la posverdad como líderes que estaban negando el cambio climático a pesar de las evidencias científicas: la política (opinión pública y narrativa de los medios) se ha desconectado casi por completo de la política legislativa (Roberts, 2010). El discurso político se articula como una herramienta independiente a la gestión, un hito comunicativo que en la apelación emocional incide en la opinión:

"no sólo hay discursos verdaderos o falsos, sino también de sentimiento y percepción" (Ramos-Chávez, 2018, p. 287)

y "no todas las opiniones son igual de verdaderas" (Abellán-Artacho, 2017, p. 12).

En la construcción de la posverdad confluyen, como señala Viner (2016):

- unos políticos que apelan constantemente a los sentimientos;

- la debilidad de los medios de comunicación, que necesitan de muchos usuarios y por tanto muchos clics para su supervivencia;

- el estilo informativo de gran parte del público que consume la información a partir de contenidos seleccionados por algoritmos.

El denominado "filtro burbuja"

"transforma de forma invisible el mundo que experimentamos mediante el control de lo que vemos y lo que no vemos" (Pariser, 2017, p. 88).

Los usuarios acceden únicamente a aquellos contenidos que tienen mayor relación con sus preferencias y aquellos que buscan informarse a través de las redes sociales terminan expuestos a un espectro de fuentes más reducido. Estas "burbujas sociales" son por tanto escenarios óptimos para la desinformación y el discurso polarizador (Nikolov et al., 2015; DiFranco; Gloria-García, 2017).

Finalmente, el ciudadano ha terminado convirtiéndose en un consumidor

"sin interés real por la política, por la configuración activa de la comunidad" (Byung-Chul, 2014, p. 13).

Los partidos buscan únicamente satisfacer a estos votantes-consumidores que desde la pasividad se acercan a la política a través de la queja y el malestar (Byung-Chul, 2014).

\section{Desinformación: el impacto de las fake news}

En la era de la posverdad, las fake news o noticias falsas emergen como una herra-

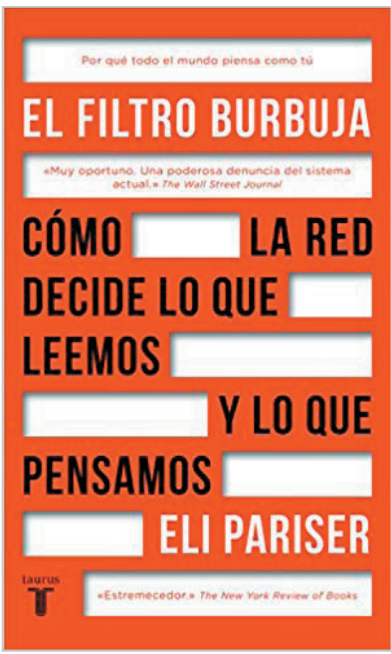

ISBN: 9788430618835 mienta para romper o reforzar los marcos de creencias, facilitando que la mentira sea más creíble que nunca. Pizarroso-Quintero señala que

"el uso de la mentira como técnica de persuasión tiene un nombre: desinformación. La propaganda no es siempre desinformación mientras que la desinformación es siempre propaganda” (Pizarroso-Quintero, 2008, p. 6).

Las noticias falsas tienen un $70 \%$ más de posibilidades de ser replicadas y llegan más lejos, más rápido y más profunda y ampliamente que la verdad en todas las categorías de información, observándose que los efectos son más pronunciados en contenidos de política que en terrorismo, desastres naturales, ciencia, leyendas urbanas o información financiera (Vosoughi; Roy; Aral, 2018). El ejemplo más notorio se recogió en la campaña por las presidenciales en EUA (2016). Las historias falsas que favorecían a Trump se compartieron 30 millones de veces, cuadruplicando la cantidad de acciones a favor de Hillary Clinton. El adulto promedio veía y recordaba 1,14 noticias falsas en este período electoral y el poder persuasivo de la desinformación fue mayor que el de otras herramientas como los spots electorales (Allcott; Gentzkow, 2017). A la alta difusión orgánica de contenido falso se añaden bots que automatizan el proceso y amplían la expansión (Shao et al., 2017), siendo la comunicación algorítmica una herramienta ampliamente utilizada (Campos-Domínguez; García-Orosa, 2018, p. 775). 
Según el estudio mundial Trust in news (Kantar Media, 2017), el 46\% de los encuestados consideraba que las noticias falsas habían influido en los resultados de las últimas elecciones. Esta percepción coincide con los resultados de las numerosas investigaciones que han abordado el uso de la desinformación como herramienta estratégica: en el referéndum por el Brexit (Narayanan et al., 2017), las elecciones presidenciales de México (Glowacki et al., 2018) y Brasil en 2018 (Machado et al., 2018) o en el plebiscito sobre el acuerdo de paz en Colombia (2016) (Parra-Valero; Oliveira, 2018), entre otros.

Frente a la tesis de Tesich (1992), gran parte de la opinión pública actual comienza a ser consciente del impacto del engaño. El 83\% de los europeos identifica la desinformación como un peligro para la democracia (Kantar TNS, 2018) y el $53 \%$ de los españoles que participaron en esta encuesta reconoció encontrarse noticias falsas todos o casi todos los días. En cuanto a la intencionalidad, el $88 \%$ de los encuestados en el estudio Influencia de las noticias falsas en la opinión pública (Estudio de Comunicación, 2018) señaló que estas informaciones tratan de perjudicar la imagen y reputación de personas u organizaciones y el 75,8\% identifica como objetivo sacar beneficio personal o para el colectivo al que pertenece.

La desinformación registra una tendencia creciente. Según un informe de Corporate Excellence (2018), en 2017 las fake news aumentaron un 365\% y la tendencia es positiva. La consultora Gartner (Panetta, 2017) prevé que en 2022 el público occidental consumirá más noticias falsas que verdaderas. Luchar contra la desinformación es ya un objetivo institucional. La Unesco ha elaborado una guía específica para periodistas (Ireton; Posetti, 2018) y la Comisión Europea (2018) ha establecido un comité de expertos formado por 39 profesionales de la política, la comunicación y la verificación que han remarcado, entre otras cuestiones, la complejidad de crear un marco legislativo en torno a este fenómeno. Ratifica esta visión la organización Freedom House (2018): en 14 países, los gobiernos que intentaron combatir las noticias falsas irónicamente terminaron restringiendo la libertad de internet.

El periodismo y la verdad que encierra su ejercicio (Marcos-Recio, 2017) son por el momento las únicas herramientas para identificar y denunciar las historias falsas. Igualmente, la verificación se ha posicionado como una nueva especialización profesional. Sólo en la última década las plataformas de fact-checking se han asentado en más de 50 países y el 90\% se ha establecido a partir de 2010 (Fernández-García, 2017).

La credibilidad de los medios de comunicación se ha visto resentida. El estudio Digital news report muestra que frente a una tendencia positiva en años anteriores, en 2018 la confianza en los medios de comunicación cayó 3 puntos. Este trabajo evidencia además la necesidad de implementar la alfabetización mediática:

"solo 3 de cada 10 usuarios en España entiende cómo decide Facebook qué noticias mostrarles en su página principal” (Amoedo; Vara-Miguel; Negredo, 2018, p. 12).

Por tanto, la comunicación política es el área que registra mayor penetración de noticias falsas y el periodismo, la verificación digital y la alfabetización mediática son por el momento las únicas vías para neutralizar la desinformación.

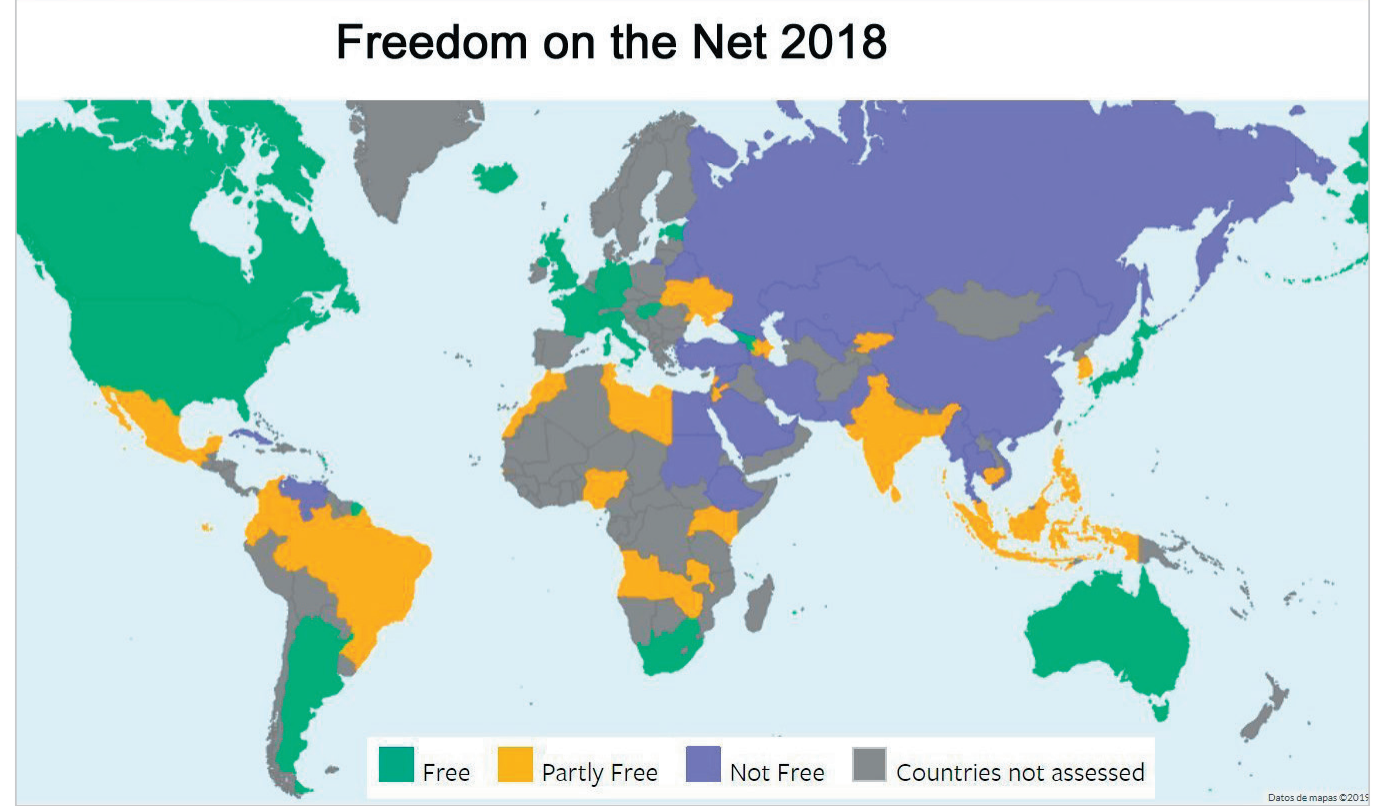

https://freedomhouse.org/report/freedom-net/freedom-net-2018 
El informe European communication monitor (2018) recoge que el 22,5\% de las organizaciones europeas se han visto afectadas por las fake news, aunque sólo el $12 \%$ ha establecido soluciones. Este nuevo contexto informativo motiva el cambio de rutinas profesionales que deben ser abordadas desde la investigación (Casero-Ripollés, 2018). Según el Anuario de la comunicación (Asociación de Directivos de Comunicación, 2018a) la posverdad será uno de los retos a superar por los profesionales de la comunicación. Igualmente, El estado de la comunicación en España (Asociación de Directivos de Comunicación, 2018b) destaca que en los próximos tres años se tendrá que abordar la regulación del uso de los datos masivos (big data) y los algoritmos en comunicación $(50,4 \%)$.

Hasta el momento, la bibliografía científica que ha recogido el impacto profesional es limitada en comparación con los estudios publicados sobre fake news y posverdad. Destaca el trabajo realizado por López-Borrull, Vives-Gràcia y Badell (2018) que empodera al profesional de la información y la documentación como especialista en la formación en alfabetización de medios y que propone, entre otras acciones, la generación de sinergias con los profesionales de la comunicación para crear proyectos en común. Este estudio tiene el propósito de recoger la visión y el papel de los profesionales de la comunicación en este nuevo contexto.

\section{Objetivos y metodología}

El objetivo general de este trabajo es abordar la desinformación desde la perspectiva profesional de distintos actores sociales para entender cómo ha impactado en sus rutinas profesionales y cuál es el grado de convivencia con este fenómeno. Para ello se realizó una investigación exploratoria que analizó la bibliografía científica relacionada con el objeto de estudio. En este primer análisis se identificaron los profesionales que tenían mayor relación con el fenómeno:

- consultores políticos, implicados en uno de los campos en los que se detecta mayor concentración de desinformación;

- periodistas, por su labor divulgadora que depende de la verificación de fuentes y la curación de contenido;

- gestores de redes sociales de medios digitales, por ser tanto emisores como receptores de la información que circula en las plataformas digitales;

- verificadores de información, nueva especialidad periodística que nace de la necesidad de esclarecer la veracidad de determinadas informaciones.

Se realizaron 24 entrevistas estructuradas vía email a una muestra profesional que recogía al menos 5 actores sociales de cada área. Se contactó a los entrevistados para explicar el objetivo del trabajo y se enviaron los cuestionarios por email, individualmente, acompañando este trabajo de seguimiento telefónico. Se optó por la entrevista por email ya que ofrece mayor espacio de reflexión, garantiza la literalidad de las respuestas en el posterior análisis y resultaba más eficaz en la orquestación del trabajo, dada la dispersión geográfica y las agendas de los participantes.

Las entrevistas estructuradas presentaban dos bloques de preguntas:

- en el primero se hacía alusión a cuestiones comunes: valoración del fenómeno, impacto en sus rutinas profesionales y posicionamiento ante una posible legislación relacionada;

- en el segundo se trataron particularidades de su campo profesional: impacto de la desinformación en su área profesional, relación con su actividad, y cambios en los procesos de monitorización de la información.

Tabla 1. Entrevista estructurada utilizada en el trabajo de campo

\begin{tabular}{|c|c|c|c|c|}
\hline \multicolumn{5}{|c|}{ Entrevista estructurada. Profesionales de la comunicación } \\
\hline & Consultores políticos & Verificadores & Responsables redes sociales & $\begin{array}{l}\text { Redactores medios } \\
\text { digitales }\end{array}$ \\
\hline \multirow{3}{*}{$\begin{array}{l}\text { Bloque de } \\
\text { preguntas (I) } \\
\text { Comunes }\end{array}$} & \multicolumn{4}{|c|}{$\begin{array}{l}\text { ¿Han alterado las fake news sus procesos de trabajo? } \\
\text { ¿Ha tenido que modificar o generar nuevos protocolos para adaptarse? }\end{array}$} \\
\hline & \multicolumn{4}{|c|}{ ¿Considera que las fake news pueden ser un fenómeno que dañe la democracia? } \\
\hline & \multicolumn{4}{|c|}{ ¿Considera necesario crear un marco legislativo para combatir la desinformación en la Red? } \\
\hline \multirow{4}{*}{$\begin{array}{l}\text { Bloque de } \\
\text { preguntas (II) }\end{array}$} & $\begin{array}{l}\text { ¿Realiza algún tipo de } \\
\text { seguimiento específico para } \\
\text { detectar bulos sobre sus } \\
\text { clientes? }\end{array}$ & $\begin{array}{l}\text { ¿Considera necesario que } \\
\text { las organizaciones realicen } \\
\text { seguimientos específicos } \\
\text { para detectar este tipo de } \\
\text { desinformación? }\end{array}$ & $\begin{array}{l}\text { ¿Realiza algún tipo de } \\
\text { seguimiento específico para } \\
\text { detectar bulos sobre su } \\
\text { empresa? }\end{array}$ & $\begin{array}{l}\text { ¿Realiza algún tipo de } \\
\text { seguimiento específico para } \\
\text { detectar bulos informativos? }\end{array}$ \\
\hline & \multicolumn{4}{|c|}{ ¿Se puede, desde la consultoría / verificación / comunicación digital / periodismo neutralizar la desinformación en internet? } \\
\hline & \multicolumn{4}{|c|}{ ¿Es la posverdad un fenómeno intrínseco de la estrategia política / la veracidad de la información / redes sociales / periodismo? } \\
\hline & $\begin{array}{l}\text { ¿Cuál es su valoración } \\
\text { sobre el impacto de la } \\
\text { desinformación y la pos- } \\
\text { verdad en la comunicación } \\
\text { política? }\end{array}$ & $\begin{array}{l}\text { ¿En qué áreas de información } \\
\text { suele detectar mayor } \\
\text { desinformación? }\end{array}$ & $\begin{array}{l}\text { ¿Cuál es su valoración sobre el } \\
\text { impacto de la desinformación } \\
\text { y la posverdad en la comuni- } \\
\text { cación digital? }\end{array}$ & $\begin{array}{l}\text { ¿Cuál es su valoración } \\
\text { sobre el impacto de la } \\
\text { desinformación y la posver- } \\
\text { dad en el periodismo? }\end{array}$ \\
\hline
\end{tabular}


Para la selección de la muestra de consultores políticos se escogieron perfiles de profesionales directivos, acostumbrados a trabajar en entornos locales, nacionales e internacionales. Se han obtenido 5 respuestas de consultores de primer nivel:

- Antoni Gutiérrez-Rubí, fundador y director de Ideograma;

- Óscar Santamaría, director de Asesores de Comunicación Pública;

- Fran Carrillo, fundador de La fábrica de discursos;

- Ángela Paloma, asesora en el Ministerio de Interior;

- Pablo Vázquez-Sande, director de Atalaya Comunicación.

La selección de los profesionales de medios de comunicación se basó en los datos de audiencia. Según el estudio de Comscore de mayo de 2018 (El mundo, 2018), el ranking de diarios digitales estaba liderado por elmundo.es, seguido por elpais.com, lavanguardia.com, ABC.es, elconfidencial.com y 20minutos.es. Encontramos cifras de audiencia similares en el estudio de AIMC (febrero-marzo 2018) que sitúa a elpais.com como el segundo sitio de internet, a lavanguardia.com como el séptimo, seguido de antena3.com en octavo lugar, dato que motivó la inclusión de este medio en el estudio.

En el caso de los periodistas se priorizaron los perfiles de las secciones de política y nacional. Han participado en este trabajo 7 profesionales (ordenados por sus respectivas audiencias):

- Iratxe Rojo, periodista de nacional y política en elmundo.es;

- Josep-María Calvet, redactor jefe de Política en lavanguardia.com;

- Jaime García-García, periodista en ABC.es;

- Paloma Esteban, redactora de política y nacional en elconfidencial.com;

- Clara Pinar, redactora de política en 20minutos.es;

- Francisco Sierra, director de Contenidos de Antena 3 Multimedia;

- Carmen Rivas-Iglesias, gerente de Informativos de Antena 3 Multimedia.

En cuanto a los responsables de redes sociales de medios de comunicación digital se ha contado con 5 participantes:

- Carmela Ríos, responsable de Nuevas Narrativas en Unidad Editorial (elmundo.es);

- Pau-Francesc Rodríguez, de La vanguardia;

- Javier Corcuera, de ABC.es;

- Eva Moreno, de El confidencial; y

- Antonio García, de antena3.com.

En relación con los verificadores, se registra un número menor de profesionales especializados, por lo que se seleccionó a:

- periodistas del fact-checker español Malditobulo.es, adherido a la International Fact-checking Network (IFCN) y participante en el grupo de especialistas seleccionado por la Comisión Europea;

- periodistas de Newtral, startup especializada en fact-checking y productora del programa El objetivo de La sexta.

Participaron 7 verificadores:

- Clara Jiménez, fundadora de Malditobulo.es;

- Inés Calderón, Argiñe Aguila, Carlos Orquín, Javier Guzmán, y Miriam Ruiz, de Newtral.

En esta muestra se recogió además el testimonio de Myriam Redondo, autora de Verificación digital para periodistas. Manual contra bulos y desinformación internacional.

El trabajo de campo se realizó entre marzo y octubre de 2018 por lo que los cargos indicados hacen alusión al momento en el que se realizó la entrevista.

\section{La desinformación según los profesionales de la comunicación. Resultados}

A pesar de que se podría presuponer que las rutinas de trabajo de los profesionales de la comunicación se han visto modificadas con la desinformación, los expertos consultados señalan en su mayoría no haber registrado cambios significativos. Las fake news no son percibidas como un fenómeno novedoso, aunque ha motivado una preocupación por parte de los clientes, en el caso de los consultores políticos, y una intensificación en los procesos de verificación en el caso de los periodistas y los gestores de redes sociales.

La desinformación se advierte como un fenómeno que puede dañar la democracia, aunque de manera espontánea cada actor social añade visiones particulares. Los consultores políticos la contextualizan en la crisis de confianza y la deslegitimación de las instituciones, los partidos políticos y los medios de comunicación; la falta de conciencia crítica de los ciudadanos y las nuevas estructuras orquestadas que ofrecen mayor viralidad y difusión a los contenidos falsos.

La deslegitimación de las instituciones es también señalada como una consecuencia que impacta en las decisiones de la ciudadanía:

"Las fake news pueden cambiar las tendencias políticas de la opinión pública e incluso desestabilizar las instituciones" (sic, verificador 03). 
Esta circunstancia propicia la polarización, que resulta un contexto social favorable para difundir bulos:

"El punto de máxima distribución de la desinformación, donde los bulos campan a sus anchas, se da cuando la población está más polarizada y por lo tanto en un momento de mayor distancia y ruptura. Un ejemplo de esto es Cataluña, el pico de desinformación para España en nuestra opinión, ya que es cuando más bulos hemos visto circular tanto del lado independentista como del constitucionalista" (sic, verificador 02).

Los profesionales de medios digitales añaden a este escenario la falta de alfabetización mediática y cierta autocrítica al referirse al clickbait y la inmediatez como factores relacionados. No obstante, se empodera el periodismo y la honestidad en su ejercicio:

"Los periodistas nos debemos a los lectores y a contar la verdad siempre, contar las historias y las noticias que el público debe saber. Nos tenemos que mantener imparciales y buscar siempre los enfoques que ayuden a la gente a entender lo que pasa. A veces hay que contar noticias que desestabilizan un Gobierno, por ejemplo, o que pueden tener consecuencias importantes para la economía familiar de mucha gente" (sic, periodista 03).

Sólo 7 de los 24 entrevistados está de acuerdo con la creación de un marco legislativo relacionado. La mayoría se muestran reacios ya que podría mermar el derecho a la información, quedar obsoleto en poco tiempo o ser mal aplicado en su práctica. También se observa la posibilidad de actualizar el marco actual y se demanda en este punto la implicación de las empresas tecnológicas:

“El periodismo ha de ser el agente fiscalizador del poder político. Toda regulación acaba funcionando contra la libertad de expresión, como los casos de periódicos ecuatorianos o venezolanos en los mandatos de Correa y Maduro-Chávez" (sic, redes sociales 01).

"Lo complicado es crear ese marco legislativo y dilucidar qué son fake news o informaciones sesgadas ideológicamente. En teoría es sencillo, en la práctica no tanto" (sic, consultor político 01).

Como alternativa se insiste en la alfabetización mediática, la cultura crítica, la autorregulación de los medios de comunicación y la necesidad de hacer buen periodismo.

Tras esta primera fotografía del fenómeno, el segundo bloque de preguntas buscaba identificar el impacto concreto de la desinformación sobre cada actividad y las soluciones aportadas por los profesionales. Los consultores políticos insisten en la necesidad de aumentar los seguimientos y estar preparados ante una posible crisis derivada:

"Se ha aumentado la monitorización, también la preocupación de algunos clientes. Y se han preparado protocolos de comunicación de crisis ante fake news" (sic, consultor político 01).

Esta circunstancia se repite en los periodistas y responsables de redes sociales, quizá el colectivo profesional más sensible por la dependencia de fuentes externas en el desarrollo de su trabajo. Sólo uno de los periodistas y dos de los gestores de redes destaca que su empresa le ha proporcionado formación específica o está implementando nuevas tecnologías (blockchain) para ser más rápidos en la verificación de información.

Para hacer frente a un volumen mayor de bulos se ha intensificado la atención en los procesos de verificación tradicionales, en el seguimiento de información y en las menciones en redes sociales; y se recurre a la consulta de los contenidos publicados por verificadores profesionales, altamente valorados por el resto de actores sociales. Se percibe preocupación en el desarrollo del trabajo, que requiere de mayor exhaustividad ya que una mala decisión en la elección de un contenido informativo puede suponer perder el prestigio del medio.

En el caso de los verificadores se han actualizado algunos protocolos ya que las trampas son cada vez más audaces:

"Ha habido que refinar esos protocolos porque ahora hay más contenidos falsos y algunos engaños son muy refinados. Hay por ejemplo vídeos basados en inteligencia artificial en los que parece que una persona que habla ha dicho algo que no ha dicho en realidad" (sic, verificador 01).

Todos los profesionales identifican vías, desde su trabajo, para luchar contra la desinformación. Los consultores políticos mencionan la ética y la responsabilidad individual en su desempeño. Desde los medios de comunicación digitales se defiende el periodismo y la verificación, aunque se insiste en que es un trabajo que debería ser realizado únicamente por especialistas y se señala la curación de contenidos de los gestores de redes sociales como labor fundamental. La verificación sirve para neutralizar la desinformación siempre que no haya alcanzado su pico de máxima difusión, ya que entonces, aunque la información sea desmentida, es complicado llegar a la audiencia que ha sido impactada.

Los entrevistados identifican posverdad como sinónimo de mentira, un juego dialéctico que lleva a la trampa. Se rechaza el uso del término y ningún profesional vincula el concepto a su área de actividad:

"Me temo que entre los periodistas también hay quien, como entre la población general, está muy cómodo 
con las mentiras que justifican una determinada ideología, que en el caso de los medios también respaldan una estrategia empresarial. Otras veces, por las prisas no se verifican las cosas. O por la falta de experiencia de profesionales recién llegados que no están adecuadamente supervisados. Lo único bueno de todo esto es que creo que deben ser muy muy pocos quienes desinforman conscientemente, que saben lo que están haciendo, lo cual no es ningún consuelo" (sic, periodista 07).

“No comparto el concepto posverdad. Creo que las noticias son verídicas o no, es decir, verdaderas o falsas. Creo que las noticias falsas son falsas, no fake ni posverdad y es así como hay que referirse a ellas. Y, por supuesto, creo que han existido siempre, pero ahora tienen un alcance potencial mucho mayor gracias a las redes sociales" (sic, verificador 04).

Al consultar por el impacto de la desinformación en la profesión, los consultores políticos inciden en la necesidad de comunicar mejor y detectar campañas orquestadas:

"Tenemos una crisis de liderazgos, de mensaje y de profesionalización. Gestionar la desinformación cuando aún no se sabe comunicar sería un suicidio en puerta propia" (sic, consultor político 05).

Los profesionales del ámbito digital se centran en la urgencia de aplicar soluciones, como la formación, para atajar el problema:

"Los expertos en comunicación digital no pueden vivir ajenos a esta realidad, necesitan ser conscientes de la gravedad del fenómeno y recibir formación específica para verificar información y combatir bulos" (sic, redes sociales 02 ).

Finalmente, y en consonancia con los resultados de investigaciones anteriores (Vosoughi; Roy; Aral, 2018), los verificadores entrevistados encuentran más desinformación en la política, seguida de bulos sobre inmigración en distintas situaciones como sucesos o crisis, pero siempre desde el discurso del odio, y por último salud y ciencia. Cabe destacar que valoran positivamente que las empresas realicen seguimientos para detectar bulos sobre su actividad, aunque al igual que los periodistas, advierten que la verificación no debe ser realizada por cualquier profesional.

\section{Profesionales de la comunicación. Retos ante la desinformación. Discusión y conclusiones}

Los profesionales de la comunicación deben adaptarse a los contextos sociales y tecnológicos que determinan la práctica de su ejercicio. La desinformación es hoy un tema candente que ha puesto en el punto de mira el desempeño de los consultores políticos, los medios de comunicación y los responsables de redes sociales. Se añaden los verificadores, erigidos como garantes de la veracidad, que ofrecen una nueva área de especialización al sector.

A lo largo de este trabajo se ha tratado de estudiar cómo ha influido la desinformación en la adaptación de sus rutinas profesionales. Se observa que las fake news no han motivado cambios significativos. Si bien se registra mayor monitorización y verificación, las empresas no están actualizando la formación de sus trabajadores a través de cursos o talleres especializados que les capaciten para detectar bulos, cada vez más sofisticados y tecnológicos. Aunque se trataba de una muestra de análisis pequeña, sólo un tercio de los periodistas y gestores de redes sociales consultados contaba con nuevas herramientas para luchar contra la desinformación.

Esta situación resulta ambigua y llamativa ya que se demanda la necesidad de formar a la ciudadanía, pero no se hace extensible a los profesionales, que se ven afectados por el refinamiento en el engaño y se muestran además preocupados por el impacto de su trabajo tanto en la sociedad como en sus compañías. Quizá las empresas del sector de la comunicación, fundamentalmente las vinculadas al periodismo, deberían reflexionar en este punto, y buscar soluciones que supongan un sello de diferenciación y calidad en su trabajo. La mayoría de profesionales consultados son partidarios de la

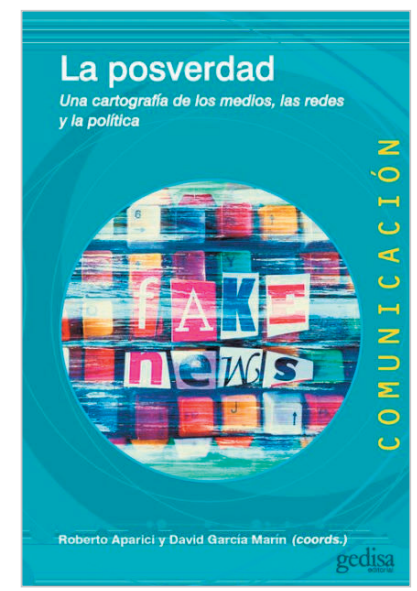

ISBN: 9788417690502 formación en verificación, y ésta contribuiría a mejorar la eficacia y tiempos dedicados a un trabajo que ahora se realiza de manera mucho más rudimentaria.

La desinformación, identificada como una amenaza para la democracia, puede suponer una oportunidad para empoderar a los profesionales de la comunicación haciendo consciente a la opinión pública de la importancia de que el flujo informativo dependa de expertos formados, capaces de hacer frente a las campañas orquestadas y a las intenciones ocultas de la información falsa. Una cadena en la que cada actor (consultores, periodistas, gestores de redes sociales y verificadores) sea garante de la honestidad y de la práctica ética, bajo el mismo paraguas de la responsabilidad, y colaborando con profesionales de otros campos (López-Borrull; Vives-Gràcia; Badell, 2018) que amplíen y consoliden el esfuerzo para luchar contra la mentira.

La falta de conciencia crítica sólo puede ser solventada mediante la alfabetización mediática. Si bien el debate tiende a centrarse en su inclusión en los planes de estu-
La falta de conciencia crítica sólo puede ser solventada mediante la alfabetización mediática 


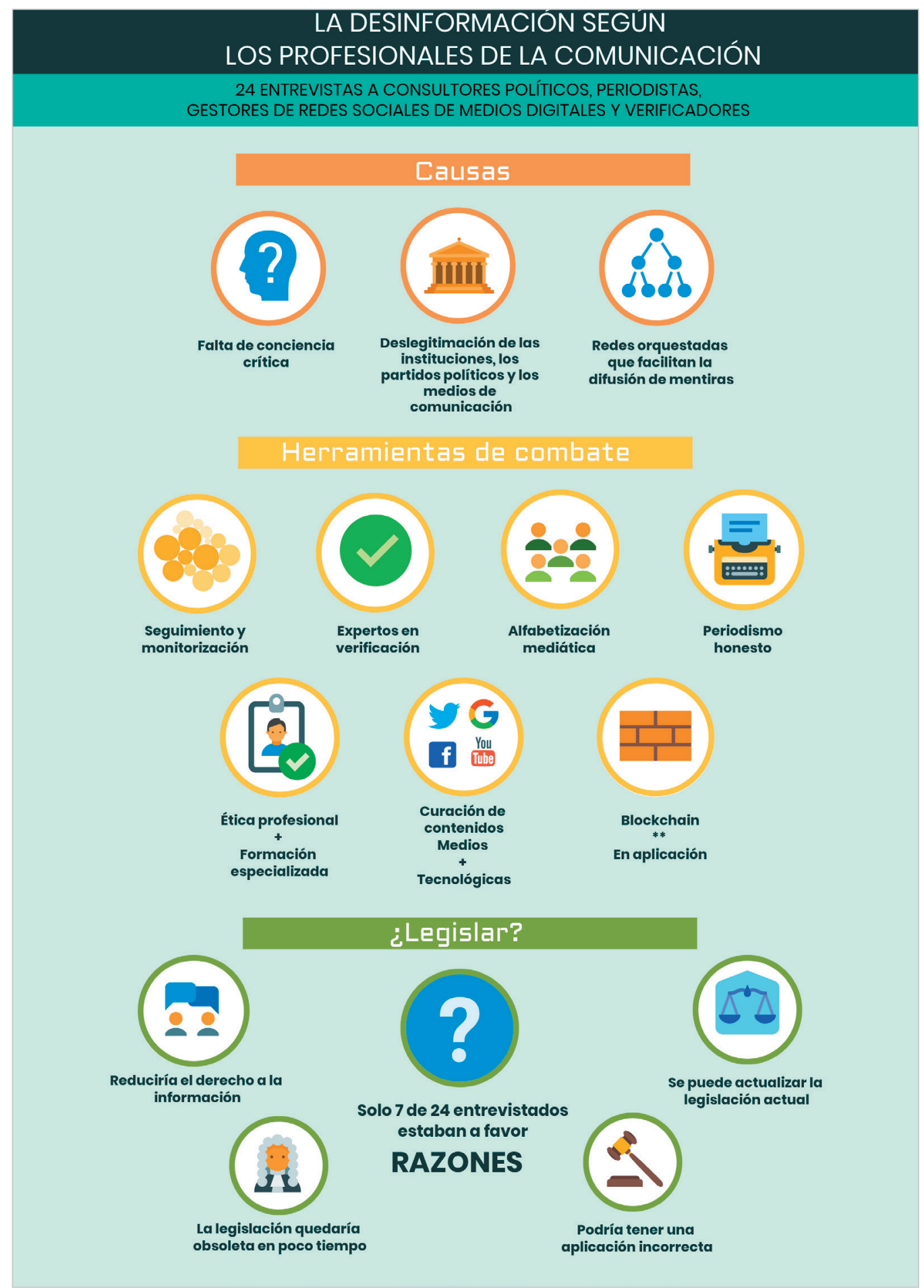

Infografía La desinformación según los profesionales de la comunicación.

dios universitarios (Rubio-Moraga; Dáder-García, 2019), su aplicación debería extenderse a otros colectivos, tan o más sensibles al consumo informativo. Esta formación debería ser asumida por profesionales de la comunicación, ampliando con ello las opciones laborales y fomentando, a través de la divulgación, la visión de la comunicación como una ciencia de valor para la sociedad.

La desinformación se encuentra en un contexto aún incipiente, por lo que se requiere de futuras investigaciones que revisen y amplíen las rutinas profesionales propuestas en este trabajo, así como las competencias, los códigos éticos y los nuevos desarrollos laborales. Así mismo, resulta de interés para futuras investigaciones relacionadas comprobar si efectivamente el uso del blockchain contribuirá a salvaguardar la veracidad de las informaciones y cómo se aplicará en los distintos ámbitos de la comunicación. En este sentido, también la verificación como campo especializado promete convertirse en un instrumento relevante, más allá del ejercicio periodístico, para salvaguardar la reputación de organizaciones e instituciones. 
Finalmente y como reflexión general, si bien, las fake news y la posverdad han generado mucho ruido mediático, parece que los pasos que estamos dando para combatirlas son lentos y de momento insuficientes. La comunicación necesita hoy más que nunca empoderarse como una ciencia de valor social, desde el ejercicio profesional y desde una divulgación que fomente una sociedad responsable y crítica con los consumos informativos.

\section{Referencias}

Abellán-Artacho, Pedro (2017). “Tiempos de posverdad: ¿qué verdades son posibles en política?”. Más poder local, v. 32, pp. 10-12.

https://dialnet.unirioja.es/servlet/articulo?codigo $=6231411$

AIMC (2018). Audiencia de internet. Febrero/marzo 2018. Asociación para la Investigación de Medios de Comunicación. https://www.aimc.es/a1mc-c0nt3nt/uploads/2018/04/internet118.pdf

Allcott, Hunt; Gentzkow, Matthew (2017). "Social media and fake news in the 2016 election". Journal of economic perspectives, v. 31, n. 2, pp. 211-236.

https://web.stanford.edu/ gentzkow/research/fakenews.pdf

Amoedo, Avelino; Vara-Miguel, Alfonso; Negredo, Samuel (2018). Digital news report.es 2018. Una audiencia diversa y preocupada por la desinformación. Universidad de Navarra.

https://www.digitalnewsreport.es

Asociación de Directivos de Comunicación (2018a). Anuario de la comunicación 2018. Madrid: Asociación de Directivos de Comunicación.

http://www.dircom.org/publicaciones/anuario/item/9153-anuario-de-la-comunicacion-2018

Asociación de Directivos de Comunicación (2018b). El estado de la comunicación en España. Madrid: Asociación de Directivos de Comunicación.

https://envios.dircom.org/docs/ECE-2018.pdf

Byung-Chul, Han (2014). Psicopolítica. Barcelona: Herder. ISBN: 9788425433993

Campos-Domínguez, Eva; García-Orosa, Berta (2018). “Comunicación algorítmica en los partidos políticos: automatización de producción y circulación de mensajes". El profesional de la información, v. 27, n. 4, pp. 769-777.

https://doi.org/10.3145/epi.2018.jul.06

Casero-Ripollés, Andreu (2018). "Research on political information and social media: Key points and challenges for the future". El profesional de la información, v. 27, n. 5, pp. 964-974.

https://doi.org/10.3145/epi.2018.sep.01

Comisión Europea (2018). Multi-dimensional approach to disinformation. Report of the independent High level Group on Fake news and Online disinformation. Directorate-General for Communication Networks, Content and Technology. https://ec.europa.eu/digital-single-market/en/news/final-report-high-level-expert-group-fake-news-and-online-disinformation

Corporate Excellence (2018). Approaching the Future 2018. Tendencias en reputación y gestión de intangibles. Corporate Excelence; Canvas; Dircom.

https://canvasconsultores.com/wp-content/uploads/2018/06/Approaching\%20the\%20Future\%202018_.pdf

DiFranzo, Dominic; Gloria-García, Kristine (2017). "Filter bubbles and fake news". XRDS: Crossroads. The ACM magazine for students, v. 23, n. 3, pp. 32-35.

https://doi.org/10.1145/3055153

El mundo (2018). "El mundo aumenta su dominio en internet". El mundo, 20 junio.

https://www.elmundo.es/television/2018/06/20/5b2a7405268e3ee7558b45c7.html

Estudio de Comunicación (2018). Influencia de las noticias falsas en la opinión pública. Estudio de Comunicación; Servimedia.

https://cutt.ly/YiOySd

https://www.servimedia.es/sites/default/files/documentos/informe_sobre_fake_news.pdf

Fernández-García, Nuria (2017). “Fake news: una oportunidad para la alfabetización mediática”. Nueva sociedad, n. 269, pp. 66-67.

https://nuso.org/media/articles/downloads/5.TC_Fernandez_269.pdf

Freedom House (2018). Freedom on the Net 2018. Manipulating social media to undermine democracy. Freedom House. https://freedomhouse.org/report/freedom-net/freedom-net-2018 
Glowacki, Monika; Narayanan, Vidya; Maynard, Sam; Hirsch, Gustavo; Kollanyi, Bence; Neudert, Lisa-Maria; Howard, Phil; Lederer, Thomas; Barash, Vlad (2018). "News and political information consumption in Mexico: Mapping the 2018 Mexican presidential election on Twitter and Facebook". The computational propaganda project. Algorithms, automation and digital politics.

https://comprop.oii.ox.ac.uk/research/working-papers/mexico2018

Ireton, Cherilyn; Posetti, Julie (2018). Journalism, fake news and disinformation. Unesco. http://unesdoc.unesco.org/images/0026/002655/265552E.pdf

Kantar Media (2017). Trust in news.

http://www2.kantar.com/l/208642/2017-10-27/6g28j

Kantar TNS (2018). "España es el país donde más encuestados se encuentran con noticias falsas de manera semanal". Blog TNSglobal.

http://blogs.tnsglobal.com/demoscopia/2018/04/casi-8-de-cada-10-espanoles-se-encuentran-con-noticias-falsas-almenos-una-vez-a-la-semana.html

Keyes, Ralph (2004). The post-truth era: Dishonesty and deception in contemporary life. New York: St. Martin's Press. ISBN: 9780312306489

Krienter, Richard (2016). "Post-truth and its consequences: What a 25-year-old essay tells us about the current moment". The nation, 30 November.

https://www.thenation.com/article/post-truth-and-its-consequences-what-a-25-year-old-essay-tells-us-about-thecurrent-moment

López-Borrull, Alexandre; Vives-Gràcia, Josep; Badell, Joan-Isidre (2018). "Fake news, ¿̇amenaza u oportunidad para los profesionales de la información y la documentación?". El profesional de la información, v. 27, n. 6, pp. 1346-1356. https://doi.org/10.3145/epi.2018.nov.17

Machado, Caio; Kira, Beatriz; Hirsch, Gustavo; Marchal, Nahema; Kollanyi, Bence; Howard, Philip N.; Lederer, Thomas; Baras, Vlad (2018). "News and political information consumption in Brazil: Mapping the first round of the 2018 Brazilian presidential election on Twitter". The computational propaganda project. Algorithms, automation and digital politics. https://comprop.oii.ox.ac.uk/research/brazil2018

Marcos-Recio, Juan-Carlos (2017). “Verificar para mejorar la información en los medios de comunicación con fuentes documentales". Hipertext.net, n. 15, p. 36-45.

https://doi.org/10.2436/20.8050.01.44

Narayanan, Vidya; Howard, Philip N.; Kollanyi, Bence; Elswah, Mona (2017). "Russian involvement and junk news during Brexit". The computational propaganda project. Algorithms, automation and digital politics.

https://comprop.oii.ox.ac.uk/research/working-papers/russia-and-brexit

Nikolov, Dimitar; Oliveira, Diego F. M.; Flammini, Alessandro; Menczer, Filippo (2015). “Measuring online social bubbles". PeerJ computer science, 1:e38.

https://doi.org/10.7717/peerj-cs.38

Oxford Dictionaries (2016). "Oxford dictionaries. Word of the year 2016 is... Post-truth".

https://www.oxforddictionaries.com/press/news/2016/12/11/WOTY-16

Panetta, Kasey (2017). Gartner top strategic predictions for 2018 and beyond. Gartner. https://www.gartner.com/smarterwithgartner/gartner-top-strategic-predictions-for-2018-and-beyond

Parra-Valero, Pablo; Oliveira, Lídia (2018). "Fake news: una revision sistemática de la literature". (OBS*) Observatorio, special issue, pp. 054-078.

https://doi.org/10.15847/obsobs12520181374

Pariser, Eli (2017). El filtro burbuja. Cómo la Web decide lo que leemos y lo que pensamos. Barcelona: Penguin Random House, Grupo Editorial SAU. ISBN: 9788430618835

Pérez, David (2017). "Posverdad, marca blanca de la mentira". ABC, 25 octubre.

http://www.abc.es/opinion/abci-posverdad-marca-blanca-mentira-201710251422_noticia.htm/

Pizarroso-Quintero, Alejandro (2008). "Justificando la guerra. Manipulación de la opinión pública en los conflictos más recientes". Revista internacional de comunicación audiovisual, publicidad y literatura, v. 1, n. 6, pp. 3-19.

https://idus.us.es/xmlui/handle/11441/57980

Ramos-Chávez, Alejandro (2018). “Información líquida en la era de la posverdad”. Revista general de información y documentación, v. 28, n. 1, pp. 283-298.

https://doi.org/10.5209/rgid.60809 
Roberts, David (2010). "Post-truth politics". Grist, 1 April. http://grist.org/article/2010-03-30-post-truth-politics

Rubio-Moraga, Ángel L.; Dáder-García, José-Luis (2019). “El futuro del periodismo en tiempos de posverdad”. En: Aparici, Roberto; García-Marín, David (eds.). La posverdad. Una cartografía de los medios, las redes y la política. Barcelona: Gedisa, pp. 2873-3129. ISBN: 9788417690502

Shao, Chengcheng; Ciampaglia, Giovanni-Lucca; Varol, Onur; Yang, Kai-Cheng; Flammini, Alessandro; Menczer, Filippo (2017). "The spread of low-credibility content by social bots". Nature communication, v. 9.

https://doi.org/10.1038/s41467-018-06930-7

Tesich, Steve (1992). "A government of lies". The nation, v. 254, n. 1, pp. 12-13.

Viner, Katharine (2016). "How technology disrupted the truth". The guardian, 12 July. https://www.theguardian.com/media/2016/jul/12/how-technology-disrupted-the-truth

Vosoughi, Soroush; Roy, Deb; Aral, Sinan (2018). "The spread of true and false news online". Science, v. 359, n. 6380, pp. 1146-1151.

https://doi.org/10.1126/science.aap9559

Zerfrass, Ansgar; Tench, Ralph; Verhoeven, Piet; Vercic, Dejan; Moreno, Ángeles (2018). European communication monitor. Communication Director.

http://www.communicationmonitor.eu/2018/06/13/ecm-european-communication-monitor-2018

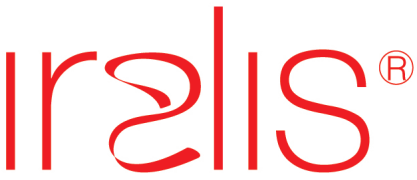

International Registry for Authors: Links to Identify Scientists

es:

- una guía para los autores hispanos para que firmen sus trabajos en el formato internacional usual

- una base de datos que registra las variantes de firma usadas por cada autor en diferentes épocas

- un buscador que usa automáticamente todas las variantes registradas

\section{El formato de firma internacional}

Con el apoyo de:

El profesional de la

\section{-información}
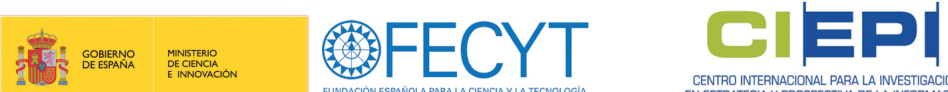

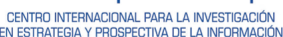

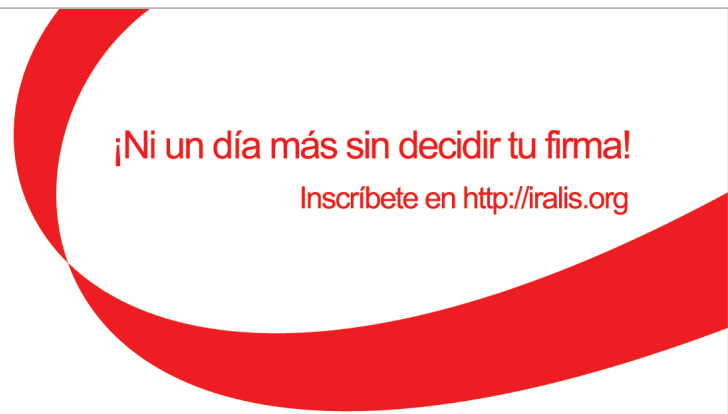

